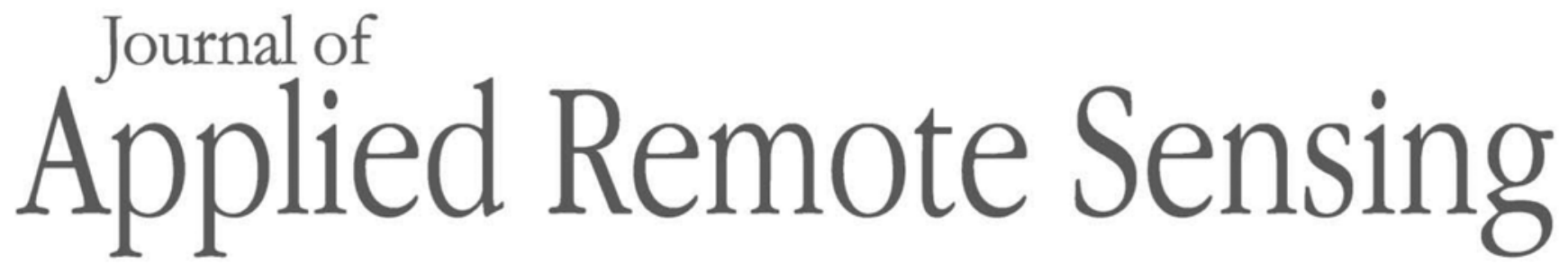

RemoteSensing.SPIEDigitalLibrary.org

\title{
On the relationship between land surface infrared emissivity and soil moisture
}

Daniel K. Zhou

Allen M. Larar

$\mathrm{Xu}$ Liu 


\title{
On the relationship between land surface infrared emissivity and soil moisture
}

\author{
Daniel K. Zhou,* Allen M. Larar, and Xu Liu \\ NASA Langley Research Center, Hampton, Virginia, United States
}

\begin{abstract}
The relationship between surface infrared (IR) emissivity and soil moisture content has been investigated based on satellite measurements. Surface soil moisture content can be estimated by IR remote sensing, namely using the surface parameters of IR emissivity, temperature, vegetation coverage, and soil texture. It is possible to separate IR emissivity from other parameters affecting surface soil moisture estimation. The main objective of this paper is to examine the correlation between land surface IR emissivity and soil moisture. To this end, we have developed a simple yet effective scheme to estimate volumetric soil moisture (VSM) using IR land surface emissivity retrieved from satellite IR spectral radiance measurements, assuming those other parameters impacting the radiative transfer (e.g., temperature, vegetation coverage, and surface roughness) are known for an acceptable time and space reference location. This scheme is applied to a decade of global IR emissivity data retrieved from MetOp-A infrared atmospheric sounding interferometer measurements. The VSM estimated from these IR emissivity data (denoted as IR-VSM) is used to demonstrate its measurement-to-measurement variations. Representative 0.25 -deg spatially-gridded monthly-mean IR-VSM global datasets are then assembled to compare with those routinely provided from satellite microwave (MW) multisensor measurements (denoted as MW-VSM), demonstrating VSM spatial variations as well as seasonal-cycles and interannual variability. Initial positive agreement is shown to exist between IR- and MW-VSM (i.e., $R^{2}=0.85$ ). IR land surface emissivity contains surface water content information. So, when IR measurements are used to estimate soil moisture, this correlation produces results that correspond with those customarily achievable from MW measurements. A decade-long monthly-gridded emissivity atlas is used to estimate IR-VSM, to demonstrate its seasonal-cycle and interannual variation, which is spatially coherent and consistent with that from MW measurements, and, moreover, to achieve our objective of investigating the relationship between land surface IR emissivity and soil moisture. (C) The Authors. Published by SPIE under a Creative Commons Attribution 3.0 Unported License. Distribution or reproduction of this work in whole or in part requires full attribution of the original publication, including its DOI. [DOI: 10.1117/1. JRS.12.016030]
\end{abstract}

Keywords: remote sensing; infrared ultraspectral sounder; surface emissivity; soil moisture.

Paper 171015 received Nov. 29, 2017; accepted for publication Feb. 9, 2018; published online Mar. 2, 2018.

\section{Introduction}

Land surface thermodynamic parameters are significant in Earth system science applications, and provide linkage to the energy balance between the land surface and atmosphere. These land surface parameters of skin temperature, emissivity, and soil moisture, together with atmospheric thermodynamic parameters, are critical to scientific research concerned with the hydrologic cycle, weather, and climate. ${ }^{1-3}$ Significant efforts have been devoted to the observation, modeling, and understanding of these parameters. ${ }^{4,5}$

Satellite-based microwave (MW) sensors have been developed and deployed to derive global soil moisture since 1978. ${ }^{6,7}$ They include passive MW sensors such as the Nimbus-7 Scanning Multichannel Microwave Radiometer (SMMR), ${ }^{8}$ the DMSP Special Sensor Microwave Imager (SSM/I), ${ }^{9}$ the TRMM Microwave Imager (TMI) ${ }^{10}$ and the Aqua Advanced Microwave Scanning Radiometer for EOS (AMSR-E), ${ }^{11}$ along with active MW sensors such as the ERS-1 and ERS-2

*Address all correspondence to: Daniel K. Zhou, E-mail: daniel.k.zhou@nasa.gov 
Scatterometer, ${ }^{12}$ and the MetOp Advanced Scatterometer (ASCAT) ${ }^{13}$ In addition, the recent missions of soil moisture and ocean salinity (SMOS) and soil moisture active passive (SMAP) are providing soil moisture measurements. ${ }^{14,15}$

Global volumetric soil moisture (VSM) products are routinely derived from satellite MW sensors. However, efforts have been made in estimating soil moisture from thermal infrared (TIR) measurements as well for many decades. ${ }^{6,16,17}$ Previous studies have also recognized that the land surface emissivity retrieved from infrared radiance measurements contains land moisture content information. ${ }^{18-21}$ In this effort, we wish to further exploit the fact that land surface water content information (i.e., soil moisture) is contained within infrared (IR) radiance measurements from the polar-orbiting environmental satellite advanced atmospheric sounding sensors, such as the Aqua Atmospheric Infrared Sounder, ${ }^{22-24}$ the MetOp Interferometer Atmospheric Sounding Instrument (IASI), ${ }^{25-27}$ and the Suomi NPP Crosstrack Infrared Sounder (CrIS). ${ }^{28-30}$

Investigation into the relationship between land surface IR emissivity and soil moisture content has been performed and reported in this paper, giving another perspective from the IR ultraspectral radiance measurements in addition to that of the traditionally used MW measurements. Global land surface emissivity with a decadal global-coverage climate variability, retrieved from satellite IR ultraspectral radiance measurements of IASI, is available to carry out this investigation. Our surface IR spectral emissivity retrieval algorithm was initially developed with data from the National Airborne Sounder Testbed-Interferometer (NAST-I). ${ }^{31}$ Emissivity is retrieved via its empirical orthogonal function amplitudes using a physical-regression retrieval; details of the retrieval algorithm are found elsewhere, ${ }^{32}$ and later, the inversion scheme, dealing with cloudy as well as cloud-free radiances has been developed and applied to IASI data to retrieve simultaneously atmospheric thermodynamic and surface or cloud microphysical parameters. ${ }^{33-35}$

The objectives of this study are to investigate the relationship between IR emissivity and soil moisture, to introduce a simple methodology to estimate VSM from land surface IR emissivity, and to intercompare the VSM estimated from the IR emissivity (hereafter denoted as IR-VSM) with that retrieved from satellite multisensor MW measurements (hereafter donated as MWVSM, obtained from the ESA CCI website). It is worth pointing out that the IR sensor detects radiation from both the atmosphere and surface. The emissivity retrieved from IR radiance is the surface emissivity, so the IR-VSM estimated is referring to a surface or near-surface soil moisture throughout this paper. Currently, a $0.25 \mathrm{deg}$ spatially-gridded monthly-mean IR land surface emissivity atlas, assembled from those data products retrieved from MetOp-A IASI measurements throughout this past decade (2007-2017), is used to estimate its corresponding IR-VSM and help characterize its seasonal-cycle and inter-annual variability. An overview of the methodology for converting IR emissivity to VSM is given in Sec. 2. IASI single field-ofview (SFOV) VSM estimation and its seasonal-cycle and interannual variations are presented in Sec. 3 and discussed in Sec. 4, followed by the conclusions drawn from them in Sec. 5.

\section{Methodology for IR-VSM Estimation}

The emissivity retrieved from satellite-measured IR radiance is an "effective" emissivity within the satellite instrument FOV, corresponding to a linear combination of pure scene types. ${ }^{34,36,37}$ Those pure scene types include water content, for example, Zhou et al. ${ }^{34}$ used this linear combination to assess satellite-retrieved emissivity for the Kalahari validation site. The laboratory measured sand emissivity is combined with other possible surface materials. The effective emissivity $\varepsilon$ can be expressed as

$$
\varepsilon=\sum_{i=1}^{n} \mathrm{a}_{i} \varepsilon_{i}
$$

where $a_{i}$ is the fractional contribution for pure scene type $i$, assuming a total of $n$ types in the FOV. Furthermore, Eq. (1) can be written as a linear combination of water emissivity and nonwater (or dry) emissivity as 


$$
\varepsilon=A \varepsilon_{\mathrm{w}}+(1-A) \varepsilon_{d}
$$

where $\varepsilon_{w}(=0.995)$ and $\varepsilon_{d}$ are wet (i.e., water) and dry (i.e., nonwater) emissivities, respectively. Coefficient $A$ is the water proportion $(0 \leq A \leq 1)$ related to the land surface soil moisture content. It is evident that retrieved emissivity is a function of soil moisture ${ }^{20,38}$ and its accuracy is critical for estimating soil moisture content.

To constrain the absolute value of VSM in our inversion processing from IR emissivity, Eq. (2) is expressed as

$$
\varepsilon=f \varepsilon_{w}+(1-f) \eta
$$

where $f$ is an empirical constraining function of VSM denoted as $\gamma$ hereafter and defined as

$$
f(\gamma)=0.5 \log \left[\frac{\log (0.501)-\log (0.50-\gamma)}{\log (0.501)-\log (\gamma)}\right] .
$$

The inversion of this function (i.e., VSM) is constrained between zero and $0.50\left(\mathrm{~m}^{3} / \mathrm{m}^{3}\right)$. The upper boundary is based on the soil moisture climatology derived from MW measurements of multiple satellite sensors, ${ }^{7,39,40}$ the maximum value of MW-VSM is found to be $<0.465$ $\left(\mathrm{m}^{3} / \mathrm{m}^{3}\right)$ where $\eta$ in Eq. (3) is defined as a pseudo dry-emissivity climatology

$$
\eta=\frac{\varepsilon^{c}-\varepsilon_{w} f\left(\gamma^{c}\right)}{1-f\left(\gamma^{c}\right)}
$$

which can be derived from a land surface model or realistic climatology from historical measurements of surface emissivity and soil moisture. Since $0.25 \mathrm{deg}$ spatially-gridded monthlymean datasets for IR emissivity and MW soil moisture climatology from satellite measurements are available, we use these two climatology datasets to derive pseudo dry-emissivity using Eq. (5), where the superscript $c$ is denoted for climatology. To calculate $\eta$, we need monthly mean VSM climatology $\left(\gamma^{c}\right)$ and monthly mean IR emissivity climatology $\left(\varepsilon^{c}\right)$. In this work, we used $\gamma^{c}$ of the global essential climate variables soil moisture dataset ${ }^{7,40,41}$ from November 1978 to December 2015; and $\varepsilon^{c}$ of IASI emissivity atlas ${ }^{34}$ from June 2007 to May 2016. It is noted that the absolute scaling constraint is included herein since the climatological data of MW-VSM and IR emissivity were used. This controls absolute scaling of the IR results but allows IR-VSM to characterize geophysical field spatial and temporal variability from the observed IR emissivity.

Emissivity at $1240 \mathrm{~cm}^{-1}(8.0645-\mu \mathrm{m}$ wavelength) is used since this spectral location has a significant emissivity sensitivity to surface water content variations, ${ }^{42}$ however, emissivity at another comparable frequency could be used as well. Hereafter, the emissivity $\varepsilon$ refers to a real total emissivity at $1240 \mathrm{~cm}^{-1}$. It is noted that pseudo dry-emissivity varies from location to location and time to time as the nonmoisture-related surface condition (e.g., soil texture, temperature, and vegetation cover) changes. To account for that variation, $\eta$ is currently computed with a monthly spatially-grid of $0.25 \mathrm{deg}$ latitude-longitude. As an example, Fig. 1 shows

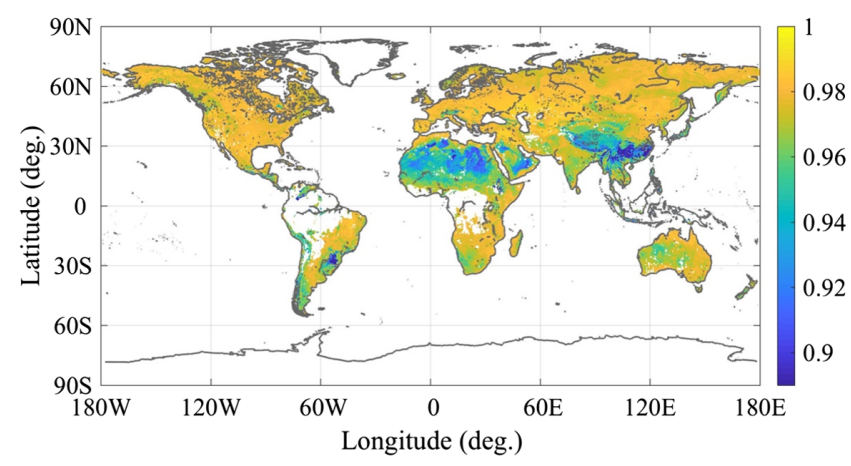

Fig. 1 A sample of pseudo dry-emissivity climatology for June is calculated using MW-VSM climatology and IR emissivity climatology at $1240 \mathrm{~cm}^{-1}$. 
a pseudo dry-emissivity climatology for the month of June. It is noted that computed pseudo dryemissivity climatology depends on time and location; in other words, the surface parameters other than real measured emissivity.

Estimation of IR-VSM is now straightforward via $f(\gamma)$ of Eq. (3) using IR-emissivity at $1240 \mathrm{~cm}^{-1}$, water emissivity at the same frequency, and a pseudo dry-emissivity $\eta$ interpolated from its original spatial and temporal grid to the location and time of observed IR emissivity. The function $f(\gamma)$ is calculated as

$$
f(\gamma)=\frac{\varepsilon-\eta}{0.995-\eta}
$$

As shown in Eq. (6), the soil moisture $\gamma$ is a function of the pseudo dry-emissivity $\eta$ and the real total emissivity $\varepsilon$ at a certain time and location. Here, it is assumed that $\varepsilon$ has a higher frequency variation containing water content information while $\eta$ has a lower frequency background variation containing nonwater content information (see Sec. 4 for a detailed discussion with examples). In this study, the $\varepsilon$ is satellite-measurement derived and the $\eta$ is climatologically predicted. The spatial and temporal resolution of $\eta$ is desired to be similar to that of $\varepsilon$ to account for spatial and temporal variations. The IR-VSM is then obtained via the inversion of $f(\gamma)$ in Eq. (4); a precalculated lookup table for $\gamma=f^{-1}$ is used to obtain IR-VSM $\gamma$.

The correlation between $\gamma$ and $\varepsilon$ can be plotted with $\eta$ at a certain location (i.e., a 0.25 -deg grid in this case), and the correlation varies from month to month. For example, $\eta$ variations observed at two random locations are shown in Fig. 2 to show that they vary in space and time. The function $f(\gamma)$ of Eq. (6) is a function of the real emissivity $\varepsilon$ and predetermined pseudo dry-emissivity $\eta$. At a certain location and time, the estimated soil moisture varies on the dashed curve as the emissivity changes, and the dashed curve changes as the time of the year changes. Currently, the temporal resolution of $\eta$ is at each month; for a daily VSM estimation, $\eta$ is linearly interpolated between two monthly climatology data points. Although it is strongly desired to

(a) $28^{\circ} \mathrm{N}, 110^{\circ} \mathrm{E}$

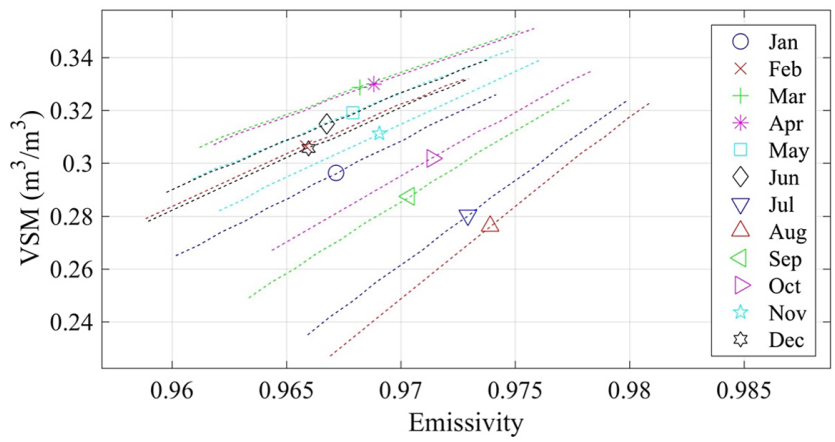

(b) $35^{\circ} \mathrm{N}, 100^{\circ} \mathrm{W}$

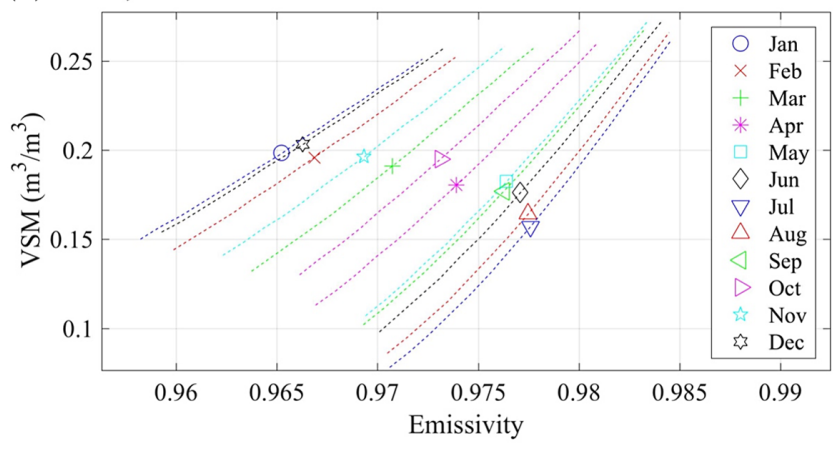

Fig. 2 Two samples of correlation between soil moisture and IR emissivity as a function of pseudo dry-emissivity depending on location and time: (a) $28^{\circ} \mathrm{N}, 110^{\circ} \mathrm{E}$ and (b) $35^{\circ} \mathrm{N}, 100^{\circ} \mathrm{W}$. The symbols shown on the curves indicate the monthly climatology of MW-VSM and IR emissivity, which are used to calculate pseudo dry-emissivity. 
have a higher temporal resolution for climatological datasets, the results herein are based on the information currently available for this study.

\section{IR-VSM Results, Variability, and Comparisons with MW-VSM}

The IR-VSM estimated by Eq. (6) is referring to surface or near-surface soil moisture. The rapid change of water content on the surface, from rain, for example, could affect estimated IR-VSM as the emissivity changes correspondingly. However, an averaged IR-VSM over a period of a few days (i.e., short term) might be able to smooth out some rapid changes of IR-VSM from high-frequency (i.e., weather)-related events and allow more of a focus on low-frequency (i.e., climatological)-related trends. This short-term-temporally averaged IR-VSM might be close to near-surface ( 0 to $5 \mathrm{~cm}$ ) VSM, as the surface and near-surface soil moisture could be similar when they are averaged. We are mindful that some differences between IR- and MW-VSM are inevitable due to the nature of the different sensor/spectral band characteristics (e.g., the effective depth of a measurement).

The pseudo dry-emissivity $\eta$ derived here has a spatial resolution of 0.25 -deg latitudelongitude (about a $25-\mathrm{km}$ diameter), and a temporal resolution of 12 months. IASI SFOV is about $12 \mathrm{~km}$, which is also the spatial resolution of retrieved $\varepsilon$. For an individual IASI measurement, we obtain a spatial-grid and linearly interpolated $\eta$ at the closest location and time of the measurement. We assume that climatological $\eta$ interpolated to that time and location of the measurement is similar to its real surface condition (e.g., surface temperature, soil texture, and vegetation coverage). Then we can use Eq. (6), the relationship between the IR-VSM and IR emissivity, to estimate IR-VSM $\gamma$ using a retrieved emissivity $\varepsilon$ and a "known" pseudo dry-emissivity $\eta$.

Daily IR-VSMs, estimated from IASI SFOV measurements, show a day-to-day (i.e., measurement to measurement) variation. Missing data are due to cloudy conditions where IR surface emissivities were not possible to retrieve. ${ }^{33,34}$ VSM depends on the measurement time and/or the situations of clouds and rain. Their day-to-day (temporal) variation is mainly from the emissivity $\varepsilon$ with "a higher frequency" variation since "a lower frequency" variation from pseudo
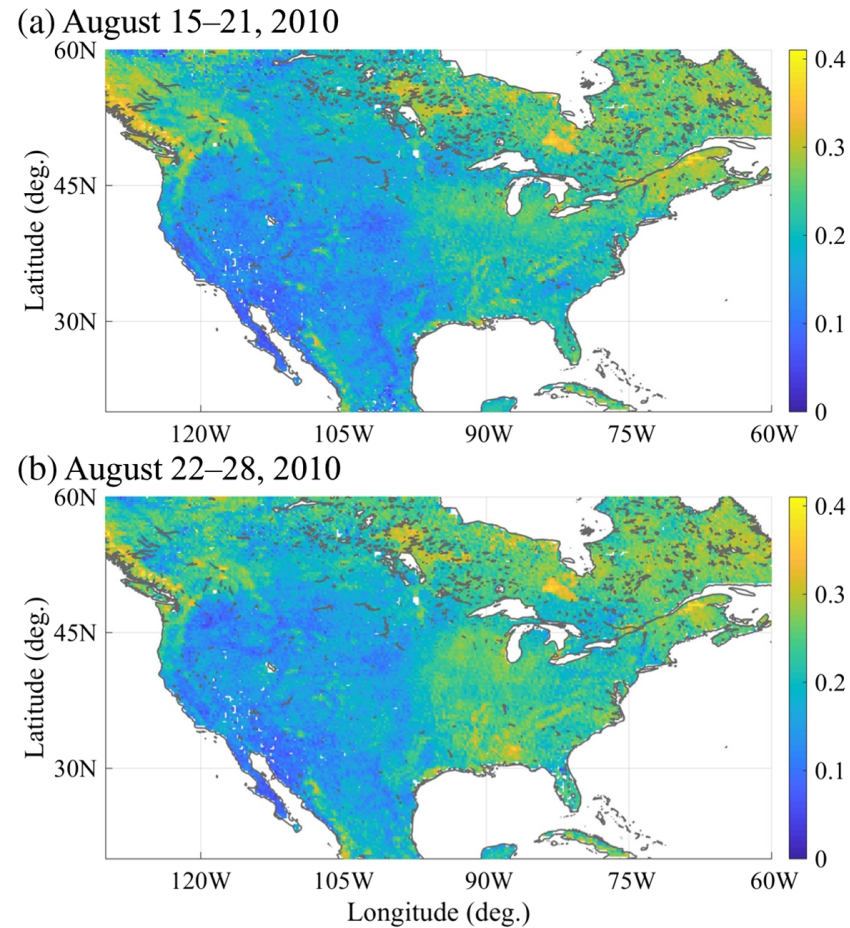

Fig. 3 IASI week-to-week soil moisture distribution from two consecutive weeks (a) August 15-21, 2010 and (b) August 22-28, 2010. 
(a) August 2010 monthly mean MW-VSM

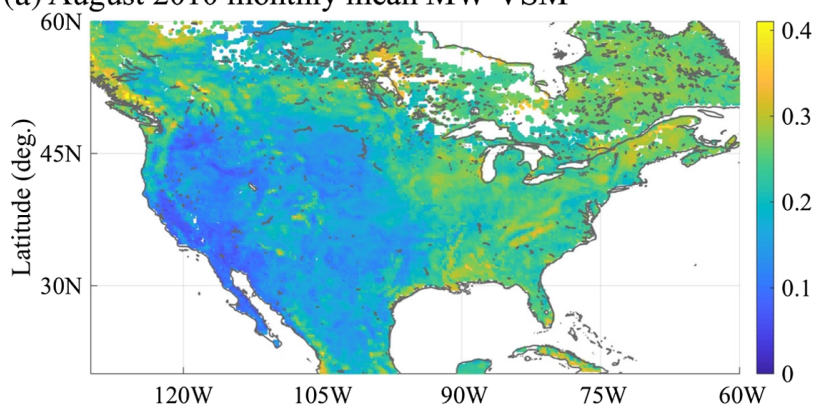

(b) August 2010 monthly mean IR-VSM

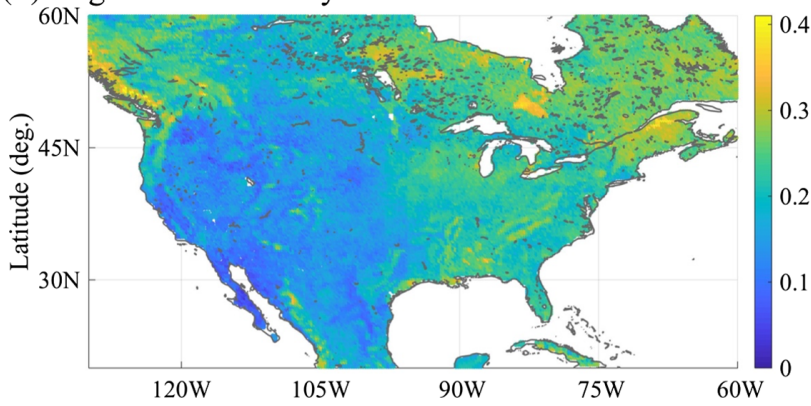

(c) Difference between (a) and (b)

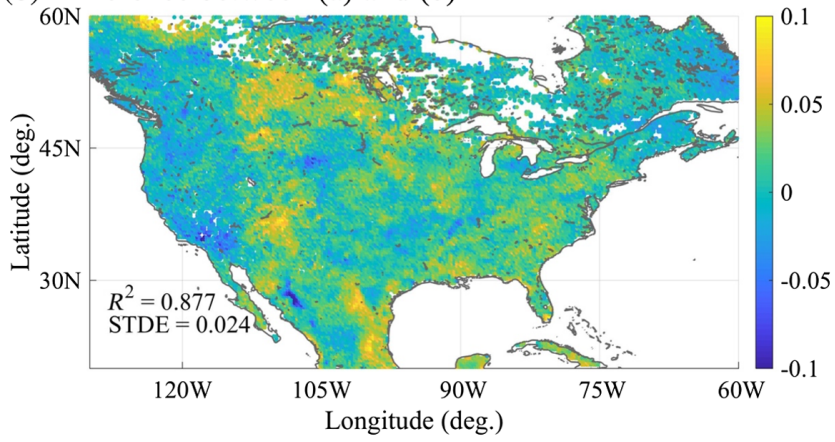

Fig. 4 August 2010 monthly mean: (a) MW-VSM, (b) IR-VSM, and (c) the difference between MW- and IR-VSM in $\left(\mathrm{m}^{3} / \mathrm{m}^{3}\right)$. The $R^{2}$ and STDE indicated are from the plotted areas.

dry-emissivity $\eta$ is assumed to be small from day to day. Figure 3 shows a 0.25 -deg weekly-mean assembled IR-VSM from two consecutive weeks of August 2010, showing the soil moisture evolution over the time from location to location. A 0.25 -deg monthly-mean assembled IRVSM is then compared with MW-VSM, shown in Fig. 4 for the same region of Northern America, as an example for August 2010; the coefficient of determination $\left(R^{2}\right)$ and the standard deviation error (STDE) are 0.877 and $0.024\left(\mathrm{~m}^{3} / \mathrm{m}^{3}\right)$, respectively. And a similar intercomparison over the globe is shown in Fig. 5 with the $R^{2}$ and STDE being 0.861 and $0.028\left(\mathrm{~m}^{3} / \mathrm{m}^{3}\right)$, respectively. It is worth pointing out that IR-VSM estimated for an individual month of a certain year (e.g., shown in Figs. 4 and 5) does use the climatological pseudo dry-emissivity of the month. Estimated IR-VSM is based on both the real emissivity (i.e., different from its climatology) and the pseudo dry-emissivity of the month (i.e., same as its climatology).

This decade-long global IR emissivity data record has already shown a seasonal-cycle and interannual variations. ${ }^{35} \mathrm{We}$ are confident that those variations should also be signified in IRVSM but wish to verify this with MW-VSM, which ensures that the corresponding IR emissivity retrieval approach is consistent. This decade long IR-VSM data record is used to examine IR-VSM seasonal-cycle and interannual variations by intercomparing them with those of MW-VSM. Many interesting phenomena are captured by VSM and are worth exploring. We have chosen two locations to demonstrate IR-VSM variability. One is the 2009 drought and 2010 greater rainfall (flooding) observed in Australia and the other is the monsoon cycle observed in South Asia. In Australia, the 2009 drought and 2010 greater rainfall (flooding) 
(a) August 2010 monthly mean MW-VSM

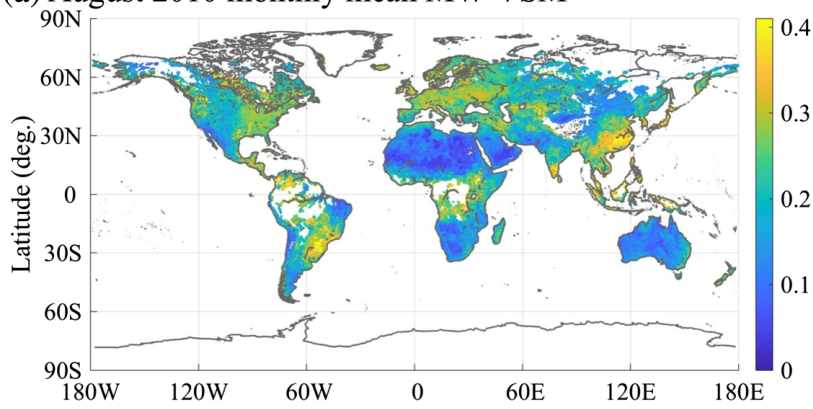

(b) August 2010 monthly mean IR-VSM

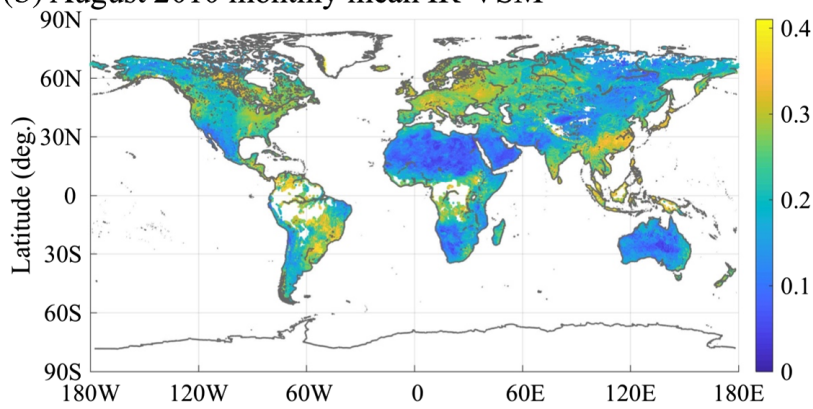

(c) Difference between (a) and (b)

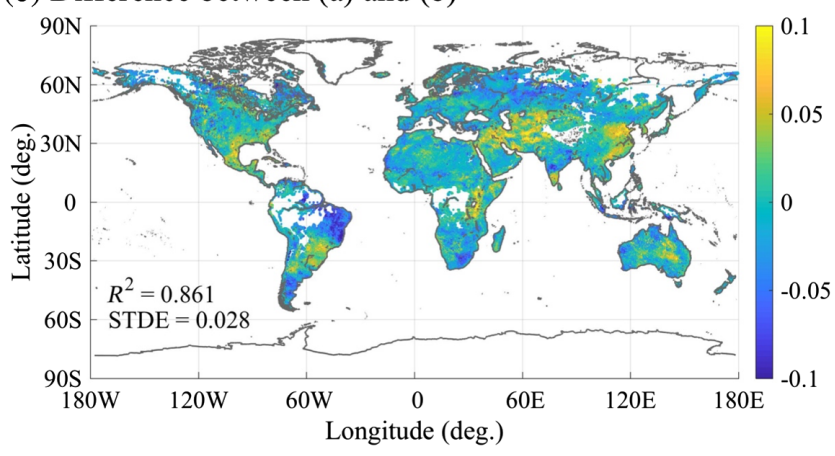

Fig. 5 Same as Fig. 4, but for a global coverage. The $R^{2}$ and STDE indicated are from the plotted areas.

are recorded during the 2009 El Niño and 2010 La Niña events, respectively. The VSM captures the drought and greater rainfall periods. Depending on the location, VSM may have seasonalcycle domination in coastal areas, whereas it may have interannual-variation domination in central regions. For example, Fig. 6 plots VSM at 23.5-deg south latitude and 135-deg east longitude showing its interannual variation captured by both MW- and IR-VSM during the 2009 El Niño and 2010 La Niña events; $R^{2}$ and STDE data shown in the plot are from the overlapping period from June 2007 to December 2016. To clearly show its interannual variation, Fig. 6(b) plots VSM for the month of September only (i.e., any random month can be selected for this demonstration). It is noted that MW-VSM is available from 1978 to 2015, but we only plotted the data from the period for which IR-VSM is available.

The second example is the monsoon cycle observed in southern Asia. In India and Pakistan, rainfall lasting from July to September is dominated by the humid southwest summer monsoon that slowly sweeps across the country beginning in late May or early June. Monsoon rains begin to recede from North India at the beginning of October. Floods and droughts are a normal occurrence in India and Pakistan; understanding the south Asian monsoon season and how it will change in the future is a fundamental challenge for climate science that has been brought to the world's attention. ${ }^{3}$ So, we have chosen a location at 25-deg north latitude and 70-deg east longitude of southeast Pakistan (near the Indian border) to reveal a seasonal cycle of VSM that is associated with the south Asian monsoon. Similar to Fig. 6, monthly mean VSM from both MWand IR-VSM showing its seasonal-cycle and interannual variations are plotted in Fig. 7(a). 
(a) $23.5^{\circ} \mathrm{S}, 135^{\circ} \mathrm{E}$ (Central Australia)

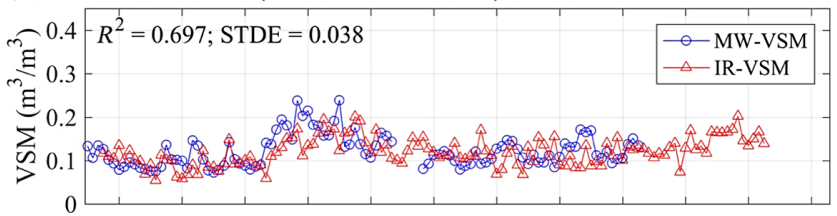

(b)

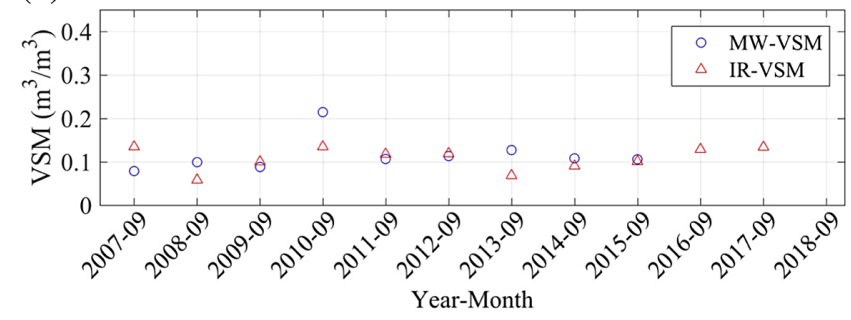

Fig. 6 (a) Monthly mean MW- and IR-VSM from central Australia, (b) September samples only. $R^{2}$ and STDE shown in the plot are from their overlapped period from June 2007 to December 2016.

(a) $25^{\circ} \mathrm{N}, 70^{\circ} \mathrm{E}$ (Southeast Pakistan)

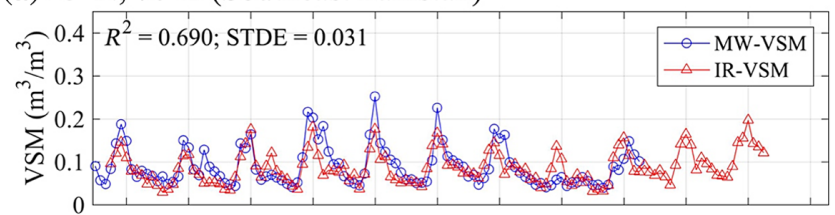

(b)

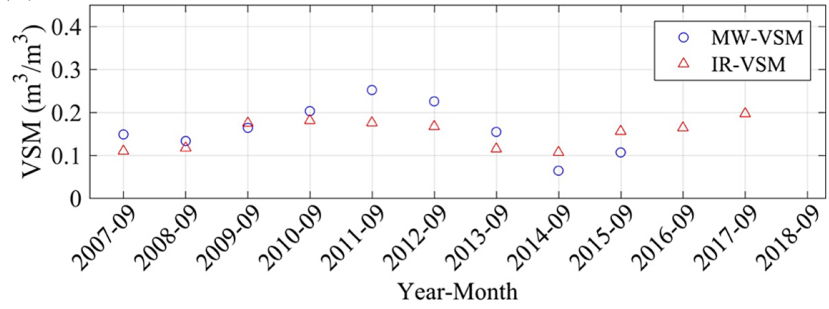

Fig. 7 Similar to Fig. 6, but from southeast Pakistan (near the Indian border).

The VSM for the month of September of each year from 2007 to 2016 is plotted in Fig. 7(b) to explain its interannual variation for clarity, showing a higher frequency variation containing water content information of $\varepsilon$ while a lower frequency background variation of $\eta$ has been set to be a climatological constant (see discussion in Sec. 4). The IR-VSM interannual variation of the same month from different years is coherent with MW-VSM, e.g., as shown in Fig. 7(b) [and Fig. 6(b)], confirming that the IR emissivity truly contains water content information.

Seasonal-cycle and interannual variation of VSM may not be obvious in all observations everywhere; it depends on surface climate and soil conditions and their spatial/temporal variability. However, the above two cases illustrate that the IR-VSM seasonal-cycle and interannual variation are practically consistent with that inferred from the MW-VSM. A few more samples from the globe showing VSM variation over the time for both MW- and IR-VSM are shown in Fig. 8 to show its seasonal-cycle and inter-annual variations at various locations. A relatively small difference is expected as they are measured by different instruments. In general, IR-VSM agrees well with MW-VSM, at least, in the sense of their variations. As shown in Figs. 4-8, a minor but significant error can be introduced by the precalculated pseudo dry-emissivity climatology and it may not precisely represent the current condition of observations at a specific time. IR-VSM accuracy is also directly dependent on the accuracy of the IR emissivity retrieval, and it is very difficult to near impossible to directly validate land surface emissivity globally. Some more discussion on IR-VSM accuracy is given in Sec. 4 to explain why the absolute accuracy of IR-VSM is not addressed in this paper. 
(a) $32.0^{\circ} \mathrm{N}, 2.4^{\circ} \mathrm{E}$ (Northern Africa)

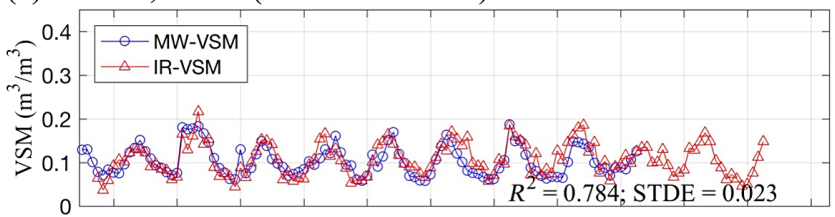

(b) $23.5^{\circ} \mathrm{N}, 76.0^{\circ} \mathrm{E}$ (India)

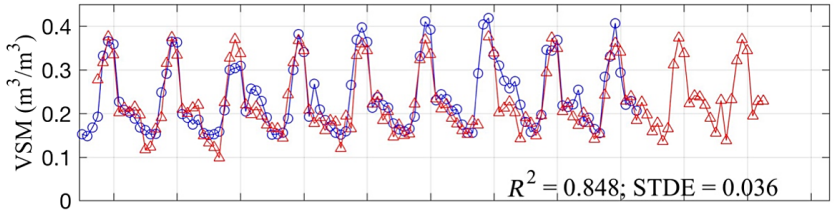

(c) $32.0^{\circ} \mathrm{N}, 112.0^{\circ} \mathrm{W}$ (Northern America)

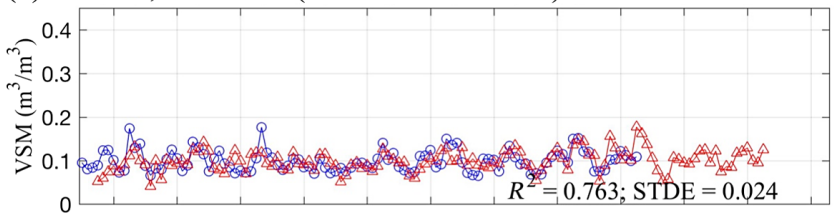

(d) $28^{\circ} \mathrm{S}, 20^{\circ} \mathrm{E}$ (Southern Africa)

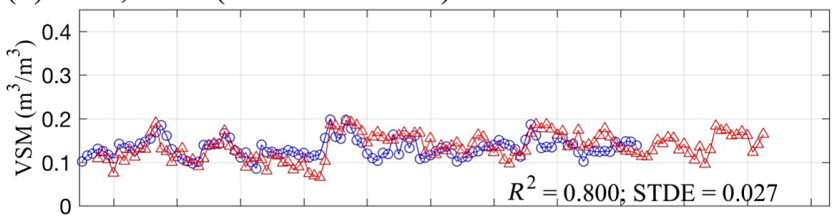

(e) $30.0^{\circ} \mathrm{S}, 119.0^{\circ} \mathrm{E}$ (Australia)

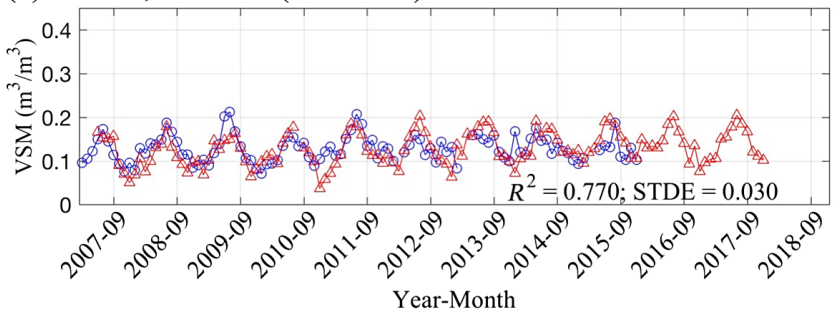

Fig. 8 Samples plotted to show VSM variation for both MW-VSM (in blue) and IR-VSM (in red) at

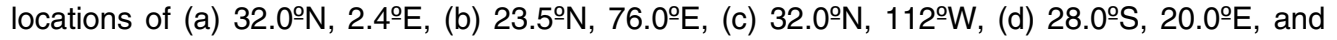
(e) $20.0^{\circ} \mathrm{S}, 119.0^{\circ} \mathrm{E}$.

\section{Discussion}

Here, we would like to use the above two cases of the 2009 drought and 2010 flooding observed in Australia and the monsoon cycle observed in south Asia to expand our discussion on Eq. (6) to investigate how soil moisture was affected by the change of the real emissivity and pseudo dry-emissivity. To carry out this discussion, soil moisture is calculated by two approaches: (1) the pseudo dry-emissivity is set to be a constant of its mean and (2) the real emissivity is set to be a constant of its mean in Eq. (6).

Figure 9 shows IR-VSM estimated with IASI emissivity and climatological pseudo dry-emissivity [same as shown in Fig. 6(a)], IR-VSM estimated with IASI emissivity and a constant pseudo dry-emissivity (scenario \#1), and IR-VSM estimated with a constant emissivity and climatological pseudo dry-emissivity (scenario \#2). A higher frequency variation from scenario \#1, relating to a real emissivity containing water content information, captures the 2009 drought and 2010 greater rainfall (flooding) events. A lower frequency background variation from scenario \#2 is only related to a climatological pseudo dry-emissivity and repeated with a period of 1 year. As mentioned above, the IR-VSM can be estimated from the real emissivity if we assume the climatological pseudo dry-emissivity is reasonably accurate. Figure 10 shows the correlation between the emissivity and IR-VSM although the monthly mean data are used. The year-to-year 


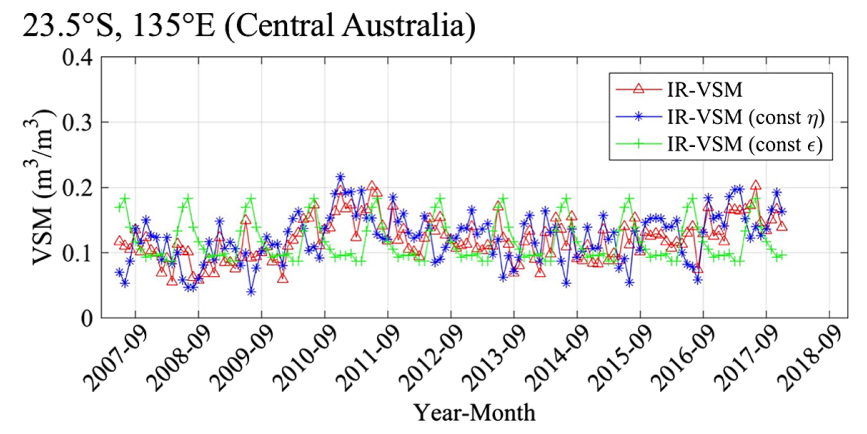

Fig. 9 IR-VSM estimated with IASI emissivity and climatological pseudo dry-emissivity (in red), IRVSM estimated with IASI emissivity and a constant pseudo dry-emissivity of 0.978 (in blue), and IR-VSM estimated with a constant emissivity of 0.964 and climatological pseudo dry-emissivity (in green, repeated every 12 months) are plotted from central Australia.

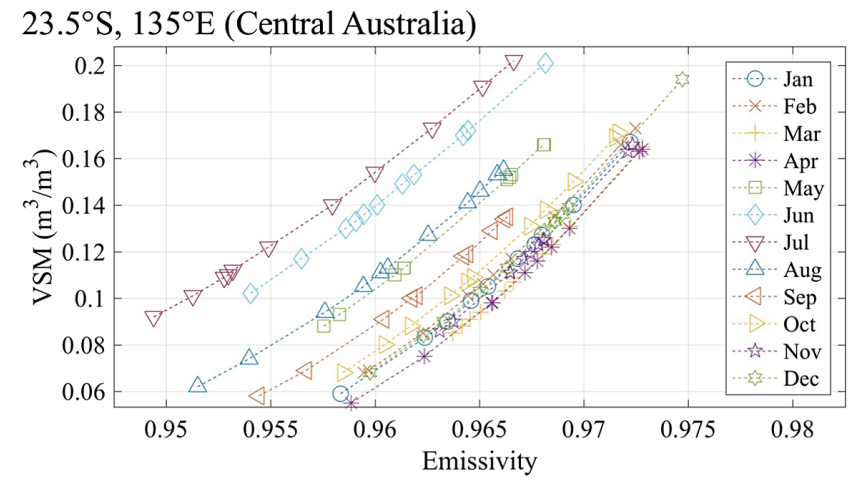

Fig. 10 Relationship between the emissivity and estimated soil moisture plotted in Fig. 6 (central Australia).

variation of IR-VSM from the same month (i.e., dashed curves of Fig. 10) depends on emissivity variation only because the climatological pseudo dry-emissivity of the month is a constant. For example, the month of July from 2007 to 2016 (i.e., a family of July monthly-mean data from 10 different years) is plotted on the dashed curve to show the relationship between IR-VSM and emissivity as described using Eqs. (4) and (6); the dashed curve is controlled by the climatological pseudo dry-emissivity of Eq. (5). IR-VSM from the same month of different years [e.g., shown in Figs. 6(b) and 10] varies as the emissivity changes, while the same climatological pseudo dry-emissivity is used.

Similar plots such as Figs. 9 and 10 are given in Figs. 11 and 12 for the second case of monsoon cycle observed in south Asia, showing the correlation between the soil moisture, emissivity, and pseudo dry-emissivity as described using Eqs. (4) and (6). As shown in Fig. 11,

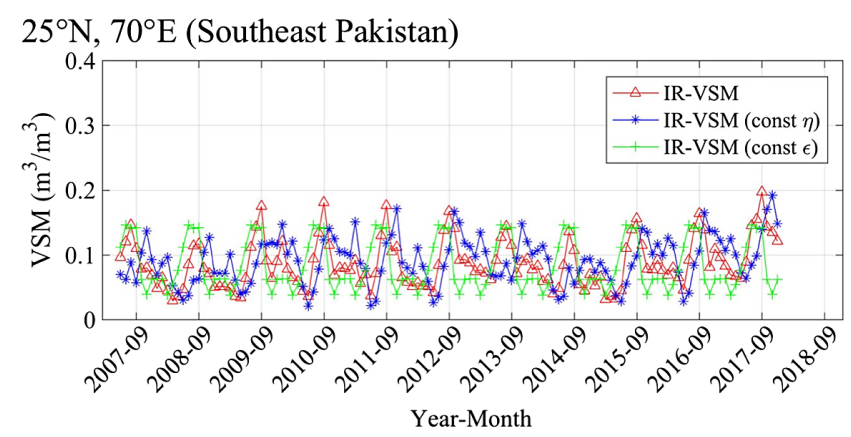

Fig. 11 Similar to Fig. 9, but from southeast Pakistan (near the Indian border): IR-VSM (in blue) uses a constant pseudo dry-emissivity of 0.980 , and IR-VSM (in green, repeated every 12 months) uses a constant emissivity of 0.962 . 
Zhou, Larar, and Liu: On the relationship between land surface infrared emissivity...

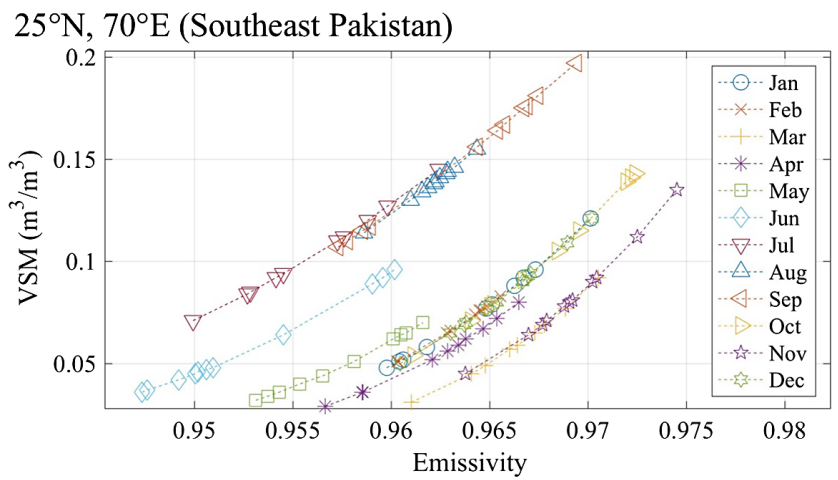

Fig. 12 Similar to Fig. 10, but from southeast Pakistan (near the Indian border).
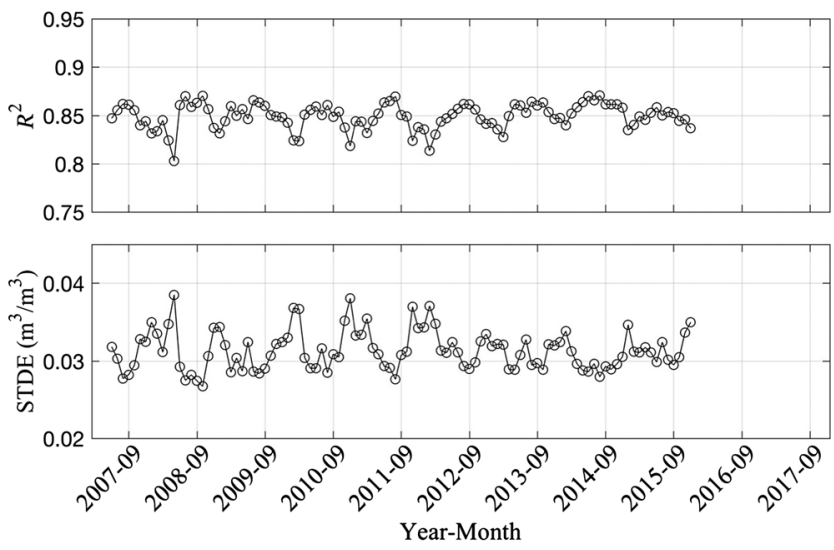

Fig. 13 Global monthly statistical parameters of $R^{2}$ and STDE between MW- and IR-VSM.

IR-VSM peaks of seasonal variation are in good agreement with MW-VSM as shown in Fig. 7(a), but they are shifted when the real emissivity or pseudo dry-emissivity is set to be a constant.

IR- and MW-VSM are not expected to be exactly the same since IR-VSM variation is affected by IR emissivity. The IR-VSM presented herein captures soil moisture variability but not the absolute accuracy, which depends on the accuracies of both emissivity and pseudo dry-emissivity. Figure 13 shows global monthly statistical parameters of $R^{2}$ and STDE between MW- and IR-VSM; each data point represents a global monthly comparison as plotted in Fig. 5. It reflects how IR-VSM compares with MW-VSM in a statistical sense for global coverage over the period from June 2007 to December 2015. Overall global $R^{2}$ and STDE during this period are estimated to be about 0.85 and $0.032\left(\mathrm{~m}^{3} / \mathrm{m}^{3}\right)$, respectively, a statistical measure between the two datasets of MW- and IR-VSM. Since the same MW-VSM climatology was employed to derive pseudo dry-emissivity used in this analysis, an overall bias between IR- and MWVSM is automatically corrected, but its STDE can still be estimated as a part of validation. As previously stated, the scope of this paper is to examine the correlation between such emissivity and soil moisture by estimating IR-VSM and comparing it with MW-VSM. We do believe that an improved IR-VSM (i.e., surface or near surface soil moisture) can be derived from IR emissivity when we have a better characterization of the pseudo dry-emissivity climatology, which depends on the accuracy of the available MW-VSM climatology.

\section{Conclusions}

In this paper, we reported on our investigation into the relationship between IR-VSM and infrared land surface emissivity from a global perspective as inferred from advanced sounder satellite data. Achieved objectives for this study were: (1) to confirm that the land surface emissivity 
retrieved from IR hyperspectral measurements contains soil moisture content information, (2) to estimate VSM using infrared land surface emissivity and climatological pseudo dry-emissivity (i.e., climatological MW-VSM and IR emissivity), and (3) to demonstrate that IR-VSM, as well as its variation, is consistent with MW-VSM using an 8-year period of overlapping satellite data from MW and IR observations.

IR-VSM is preliminarily estimated from IASI SFOV measurements and a pseudo dry-emissivity climatology. In addition to their temporal variation from week to week and month to month, the spatial distribution is found reasonable, in the sense of its monthly mean intercomparison with that derived from the MW measurements (i.e., ESA CCI VSM). A decade-long global climatology of monthly-gridded emissivity from IASI is used to estimate IR-VSM and to demonstrate its seasonal-cycle and interannual variations. Soil moisture estimated from IR emissivity and a pseudo dry-emissivity climatology is shown to be spatially coherent and consistent with that from MW measurements. IR-VSM seasonal-cycles and interannual variations are captured and verified with those of MW-VSM. Positive agreement between the IR-VSM estimated from IASI emissivity and MW-VSM indicates that IR land surface emissivity contains water content information and, when used to estimate IR-VSM, is consistent with that obtained from MW measurements. Positive results from IR- and MW-VSM intercomparisons confirm the correlation between soil moisture, IR land emissivity, and pseudo dry-emissivity as described using Eq. (6).

Our future work in this area will include obtaining or deriving a higher temporal resolution database for a climatological pseudo dry-emissivity, and validating for IR-VSM daily products with other satellite and/or in situ data. Both IR-VSM and emissivity will be routinely derived using IASI SFOV measurements from both MetOp IASI satellite sensors with a desired higher temporal-resolution climatological database of pseudo dry-emissivity. This scheme can also be applied to IR emissivity retrieved from the SNPP CrIS sensor as well as other types of satellite instruments, such as the moderate resolution imaging spectroradiometer of NASA Terra and Aqua spacecraft ${ }^{43-45}$ to estimate its land VSM from satellite IR measurements. MW-VSM data from recent and/or new satellite sensors are desired to continue these intercomparisons and/or validation of IR-VSM. Multisatellite sensor data from both IR and MW measurements might be used together to evaluate soil moisture product and provide a long-term global soil moisture climatological dataset.

\section{Acknowledgments}

This work, including the land surface emissivity retrieval algorithm, was performed under the NAST-I program supported by NASA Headquarters and NASA Langley Research Center. IASI was developed and built under the responsibility of the Centre National d'Etudes Spatiales (CNES). It is flown aboard the MetOp-A and -B satellites as part of the EUMETSAT Polar System. The IASI L1C data are received through NOAA Comprehensive Large Array-data Stewardship System (CLASS). The ESA CCI Surface Soil Moisture Product (i.e., MWVSM) was downloaded from ESA CCI Soil Moisture website. The authors wish to specifically acknowledge Dr. Jack Kaye of NASA's Science Mission Directorate for his continued, enabling support of the NAST-I program.

\section{References}

1. Z. Li et al., "Satellite-derived land surface temperature: current status and perspectives," Remote Sens. Environ. 131, 14-37 (2013).

2. A. G. Turner and H. Annamalai, "Climate change and the South Asian Monsoon," Nat. Clim. Change 2, 587-595 (2012).

3. S. I. Seneviratne et al., "Investigating soil moisture-climate interactions in a changing climate: a review," Earth Sci. Rev. 99, 125-161 (2010).

4. R. H. Reichle et al., "Global soil moisture from satellite observations, land surface models, and ground data: implications for data assimilation," J. Hydrometeorol. 5, 430-442 (2004).

5. Y. Fan and H. van den Dool, "Climate prediction center global monthly soil moisture data set at $0.5^{\circ}$ resolution for 1948 to present," J. Geophys. Res. 109, D10102 (2004). 
6. L. Wang and J. J. Qu, "Satellite remote sensing applications for surface soil moisture monitoring: a review," Front. Earth Sci. Chin. 3(2), 237-247 (2009).

7. M. Owe, R. de Jeu, and T. Holmes, "Multisensor historical climatology of satellite-derived global land surface moisture," J. Geophys. Res. 113, F01002 (2008).

8. P. Gloersen et al., "A summary of results from the first NIMBUS 7 SMMR observation," J. Geophys. Res. 89, 5335-5344 (1984).

9. J. P. Hollinger, J. L. Peirce, and G. A. Poe, "SSM/I instrument evaluation," IEEE Trans. Geosci. Remote Sens. 28(5), 781-790 (1990).

10. R. Binglish et al., "Soil moisture estimates from TRMM microwave imager observation over the Southern United States," Remote Sens. Environ. 85(4), 507-515 (2003).

11. E. G. Njoku, T. K. Chan, and S. V. Nghiem, "Soil moisture retrieval from AMSR-E," IEEE Trans. Geosci. Remote Sens. 41(2), 215-229 (2003).

12. W. Wagner, G. Lemoine, and H. Rott, "A method for estimating soil moisture from ERS scatterometer and soil data," Remote Sens. Environ. 70(2), 191-207 (1999).

13. Z. Bartalis et al., "Initial soil moisture retrievals from the METOP-A advanced scatterometer (ASCAT)," Geophys. Res. Lett. 34(20), L20401 (2007).

14. Y. H. Kerr et al., "Soil moisture retrieval from space: the soil moisture and ocean salinity (SMOS) mission," IEEE Trans. Geosci. Remote Sens. 39(8), 1729-1735 (2001).

15. D. Entekhabi et al., "The soil moisture active passive (SMAP) mission," Proc. IEEE 98(5), 704-716 (2010).

16. W. W. Verstraeten et al., "Soil moisture retrieval using thermal inertia, determine with visible and thermal spaceborne data, validated for European forests," Remote Sens. Environ. 101(3), 299-314 (2006).

17. T. N. Carlson, R. R. Gillies, and E. M. Perry, "A method to make use of thermal infrared temperature and NDVI measurements to infer surface soil water content and fractional vegetation cover," Remote Sens. Rev. 9(1), 161-173 (2009).

18. M. Urai, T. Matsunaga, and T. Ishii, "Relationship between soil moisture content and thermal infrared emissivity of the sand sampled in Muus Desert China," Remote Sens. Soc. Jpn. 17(4), 322-331 (1997).

19. K. Ogawa, T. J. Schmugge, and S. Rokugawa, "Observations of the dependence of the thermal infrared emissivity on soil moisture," Geophys. Res. Abstr. 8(4), 996 (2006).

20. M. Mira et al., "Soil moisture effect on thermal infrared $(8-13 \mu \mathrm{m})$ emissivity," IEEE Trans. Geosci. Remote Sens. 48(5), 2251-2260 (2010).

21. V. Garcia-Santos et al., "Effect of soil moisture on the angular variation of thermal infrared emissivity of inorganic soils," IEEE Geosci. Remote Sens. Lett. 11(6), 1091-1095 (2014).

22. T. S. Pagano et al., "Prelaunch and in-flight radiometric calibration of the atmospheric infrared sounder (AIRS)," IEEE Trans. Geosci. Remote Sens. 41(2), 265-273 (2003).

23. H. H. Aumann et al., "AIRS/AMSU/HSB on the aqua mission: design, science objective, data products, and processing systems," IEEE Trans. Geosci. Remote Sens. 41(2), 253-264 (2003).

24. C. Chahine et al., "AIRS: improving weather forecasting and providing new insights into climate," Bull. Am. Meteorol. Soc. 87, 911-926 (2006).

25. D. Blumstein et al., "IASI instrument: technical overview and measured performances," Proc. SPIE 5543, 196-207 (2004).

26. D. K. Klaes et al., "An introduction to the EUMETSAT polar system," Bull. Am. Meteorol. Soc. 88, 1085-1096 (2007).

27. F. Hilton et al., "Hyperspectral earth observation from IASI: five years of accomplishments," Bull. Am. Meteorol. Soc. 93, 347-370 (2012).

28. H. J. Bloom, "The cross-track infrared sounder (CrIS): a sensor for operational meteorological remote sensing," in Fourier Transform Spectroscopy, OSA Trends in Optics and Photonics, Vol. 3, pp. 1341-1343, Optical Society of America (2001).

29. L. L. Strow et al., "Spectral calibration and validation of the cross-track infrared sounder on the Suomi NPP satellite," J. Geophys. Res. 118, 12486-12496 (2013).

30. M. Divakarla et al., "The CrIMSS EDR algorithm: characterization, optimization, and validation," J. Geophys. Res. 119, 4953-4977 (2014). 
31. D. Cousins and W. L. Smith, "National polar-orbiting operational environmental satellite system (NPOESS) airborne sounder testbed-interferometer (NAST-I)," Proc. SPIE 3127, 323-331 (1997).

32. D. K. Zhou et al., "Thermodynamic product retrieval methodology for NAST-I and validation," Appl. Opt. 41(33), 6957-6967 (2002).

33. D. K. Zhou et al., "All weather IASI single field-of-view retrievals: case study-validation with JAIVEx data," Atmos. Chem. Phys. 9(6), 2241-2255 (2009).

34. D. K. Zhou et al., "Global land surface emissivity retrieved from satellite ultraspectral IR measurements," IEEE Trans. Geosci. Remote Sens. 49(4), 1277-1290 (2011).

35. D. K. Zhou, A. M. Larar, and X. Liu, "MetOp-A/IASI observed continental thermal IR emissivity variations," IEEE J. Sel. Top. Appl. Earth Obs. Remote Sens. 6(3), 1156-1162 (2013).

36. E. Valor and V. Caselles, "Mapping land surface emissivity from NDVI: application to European, African, and South American areas," Remote Sens. Environ. 57(3), 167-184 (1996).

37. G. C. Hulley et al., "Validation of the atmospheric infrared sounder (AIRS) version 5 land surface emissivity product over Namib and Kalahari deserts," J. Geophys. Res. 114, 9104.1-9104.11 (2009).

38. J. M. Sánchez et al., "Thermal infrared emissivity dependence on soil moisture in field conditions," IEEE Trans. Geosci. Remote Sens. 49(11), 4652-4659 (2011).

39. R. A. M. de Jeu et al., "A spatially coherent global soil moisture product with improved temporal resolution," J. Hydrol. 516, 284-296 (2014).

40. Y. Y. Liu et al., "Developing an improved soil moisture dataset by blending passive and active microwave satellite-based retrievals," Hydrol. Earth Syst. Sci. 15(2), 425-436 (2011).

41. Y. Y. Liu et al., "Trend-preserving blending of passive and active microwave soil moisture retrievals," Remote Sens. Environ. 123, 280-297 (2012).

42. J. W. Salisbury and D. M. D'Aria, "Emissivity of terrestrial material in the 8-14 $\mu \mathrm{m}$ atmospheric window," Remote Sens. Environ. 42, 83-106 (1992).

43. Z. M. Wan and Z.-L. Li, "A physics-based algorithm for retrieving land-surface emissivity and temperature from EOS/MODIS data," IEEE Trans. Geosci. Remote Sens. 35(4), 980996 (1997).

44. V. García-Santos et al., "Analyzing the anisotropy of thermal infrared emissivity over arid regions using a new MODIS land surface temperature and emissivity product (MOD21)," Remote Sens. Environ. 169, 212-221 (2015).

45. S. W. Seemann et al., "Development of a global infrared land surface emissivity database for application to clear sky sounding retrievals from multi-spectral satellite radiance measurements," J. Appl. Meteorol. Climatol. 47, 108-123 (2008).

Daniel K. Zhou is currently a physical scientist within the science directorate of NASA Langley Research Center. He received his PhD in physics from Utah State University, Logan, Utah, in 1992. His experience includes retrieval algorithm development for surface and atmospheric thermodynamic parameters and trace gas profiles from remotely sensed infrared measurements, as well as retrieval analysis and validation. He has received numerous awards including the NASA Exceptional Achievement Medal.

Allen M. Larar received his $\mathrm{PhD}$ in atmospheric and space sciences from the University of Michigan, Ann Arbor, in 1993. Currently, he is a senior research scientist within the chemistry and dynamics branch of the science directorate at NASA Langley Research Center in Hampton, Virginia. He has over 25 years of experience in advancing passive remote sensing measurement capabilities focusing on remote sensing of atmospheric thermodynamic state and trace species composition.

Xu Liu received his PhD from the University of Denver, Denver, Colorado, in 1989. Currently, he is a senior research scientist at science directorate, NASA Langley Research Center. His research interests include fast radiative transfer model and retrieval algorithm development for both passive and active remote sensing instruments ranging from microwave to visible spectral range. He received his numerous awards, such as NASA inventions and contributions board awards, and NASA exceptional achievement medal. 